\title{
Polyplexes Are Endocytosed By and Trafficked Within Filopodia
}

\author{
Nilesh P. Ingle, Joseph K. Hexum, and Theresa M. Reineke* \\ Department of Chemistry, University of Minnesota, Minneapolis, MN 55455.
}

$\underline{\text { Supporting Information }}$ 
Table of Contents

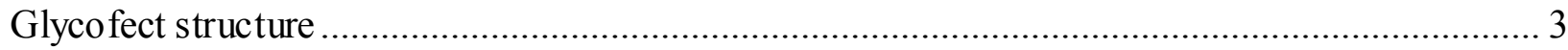

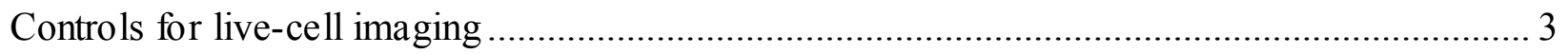

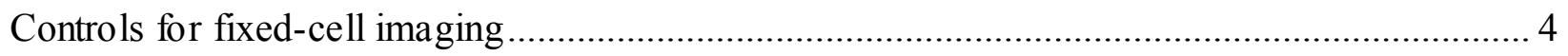

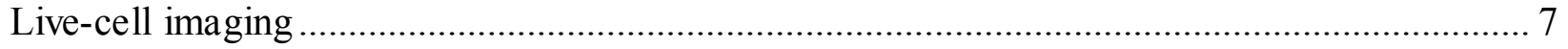

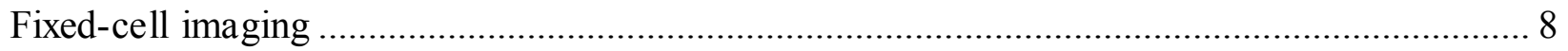

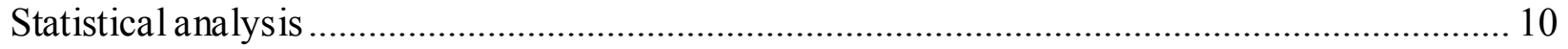

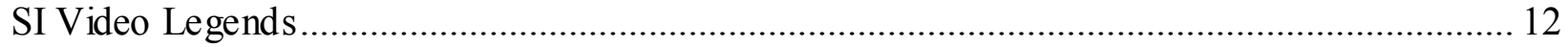

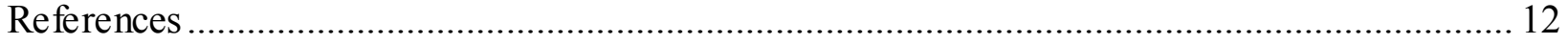




\section{Glycofect structure}<smiles>[R]CNCC(C)(C)NC(=O)[C@@H](O)[C@H](O)[C@@H](O)[C@H](O)[C@H](O)C(C)=O</smiles>

Figure S1. Structure of Glycofect ${ }^{\mathrm{TM}}$ polymer. $^{1-5}$

\section{Controls for live-cell imaging}

Live-cell imaging was performed with the objective to observe differential interference contrast (DIC) images of filopodia at high resolution. Köhler illumination was carefully calibrated and maximum extinction was achieved for DIC using a 60x objective. Next, a 100x objective was used for imaging. In order to minimize focal drift, the stage Delta $\mathrm{T}$ dish was allowed to equilibrate to $37^{\circ} \mathrm{C}$ using the temperature control.
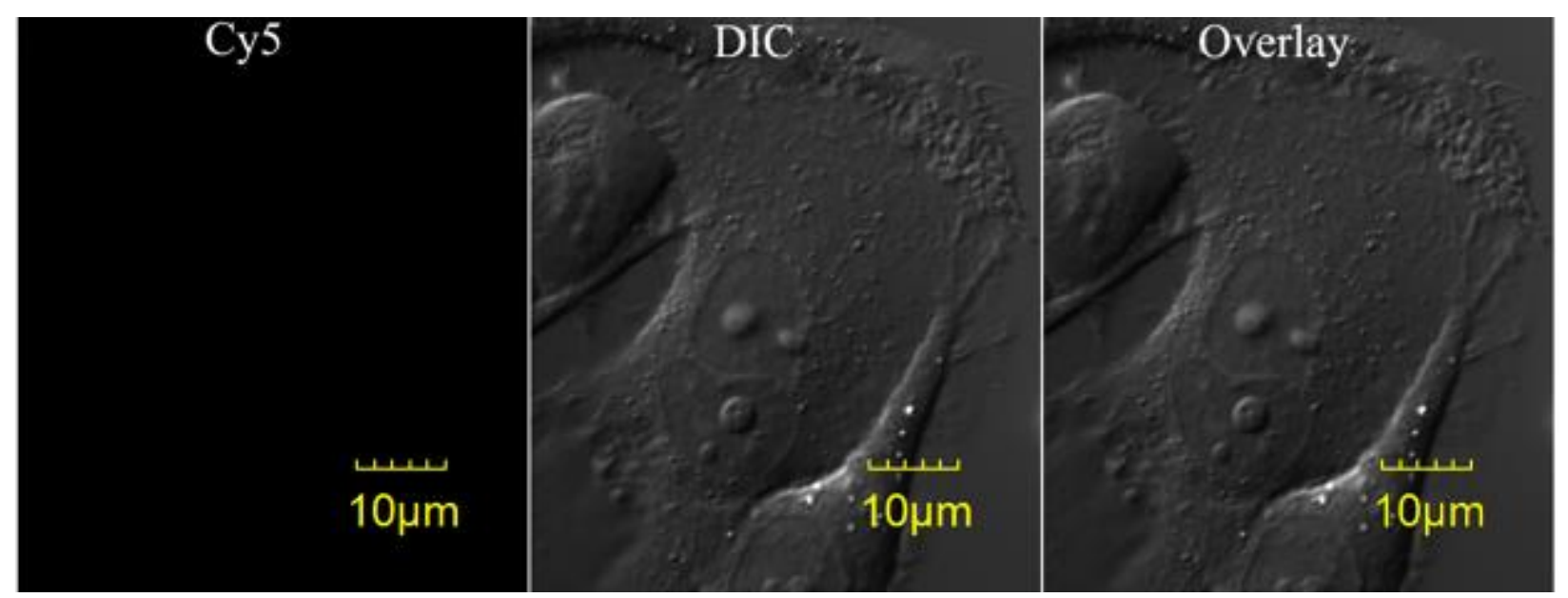

Figure S2. HeLa-Actin-GFP cells only control for live-cell imaging. The $633 \mathrm{~nm}$ laser was used to image both the Cy5-channel and the DIC-channel. The $488 \mathrm{~nm}$ laser was not used and the GFP channel was not utilized for the live cell imaging in this figure. Confocal microscope: Olympus FluoView FV1000 IX2. Objective: 60x, NA: 1.2, oil immersion. 


\section{Controls for fixed-cell imaging}

Controls for fixed-cell imaging are shown in Figures S3-S4. In these images, the confocal microscope was setup to capture clear DIC images of filopodia. The GFP channel was also utilized for fixed-cell imaging. However the GFP signal was too dim to visualize in many of the images. 


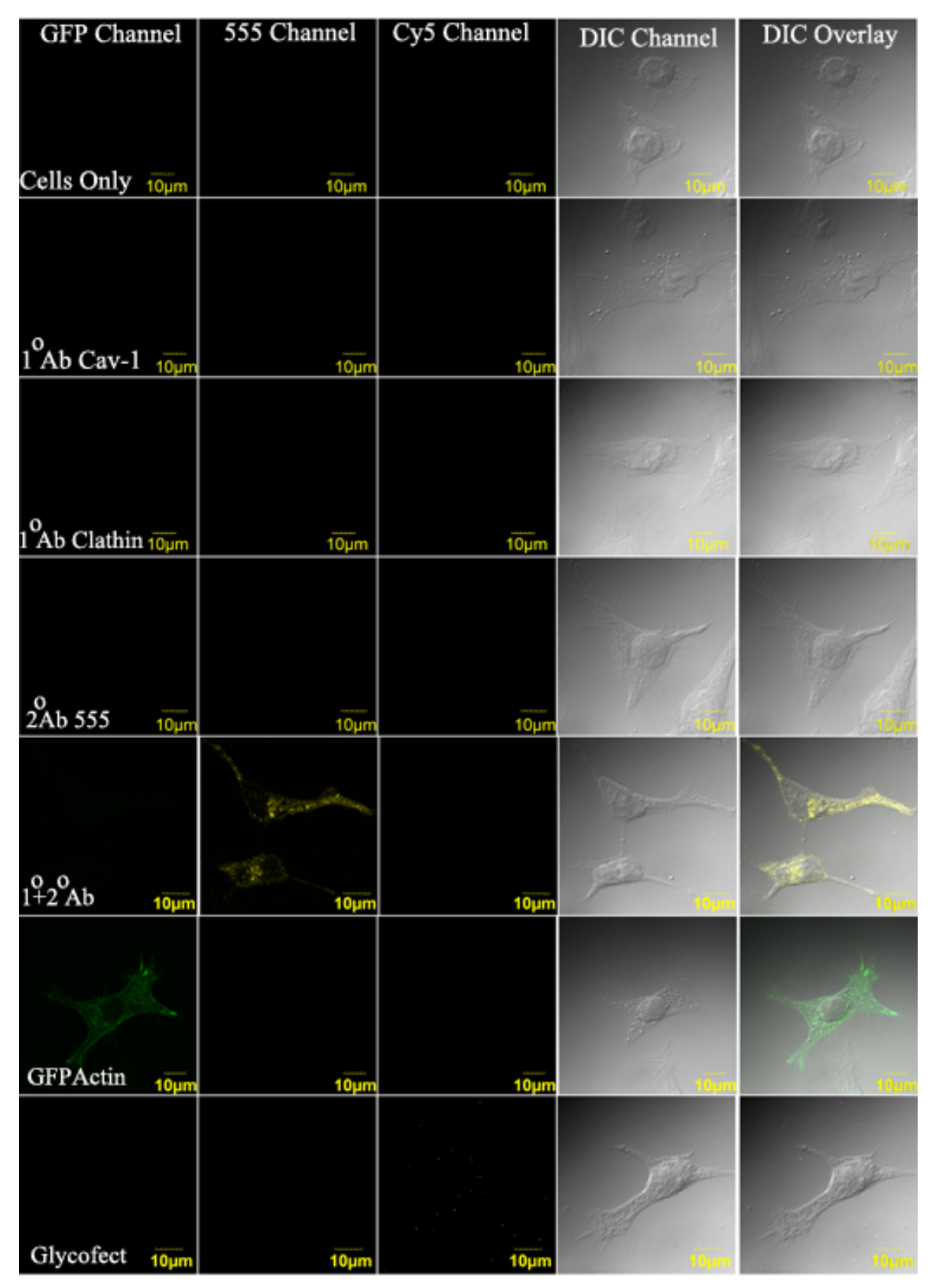

Figure S3. Controls for fixed cell imaging using sequential imaging. All controls were in HeLa cells, except GFP Actin control was in HeLa-Actin-GFP cell. Cells only: untreated HeLa cells. $1^{\circ} \mathrm{Ab}$ cav-1: primary antibody against caveolin- 1 only. $1^{\circ} \mathrm{Ab}$ clathrin: primary antibody against clathrin. $2^{\circ} \mathrm{Ab} 555$ : secondary antibody 555 only. $1^{\circ}+2^{\circ} \mathrm{Ab}$ : primary antibody clathrin and secondary antibody 555 (pseudo colored yellow). GFP Actin: HeLa-Actin-GFP cells stably expressing GFP-actin (pseudo colored green). Glycofect: HeLa cells were treated (i.e. transfected) with Glycofect polyplexes. These polyplexes were made by adding Glycofect polymer at $20 \mathrm{~N} / \mathrm{P}$ to fluorescently labeled plasmid Cy5-pDNA at $0.02 \mu \mathrm{g} / \mu \mathrm{L}$ (magenta color). Confocal microscope: Olympus FluoView FV1000 IX2. Objective: 100x, NA: 1.3, oil immersion. Excitation lasers: $488 \mathrm{~nm}$ for GFP, $543 \mathrm{~nm}$ for Alexa Fluor 555, $633 \mathrm{~nm}$ for Cy5. 


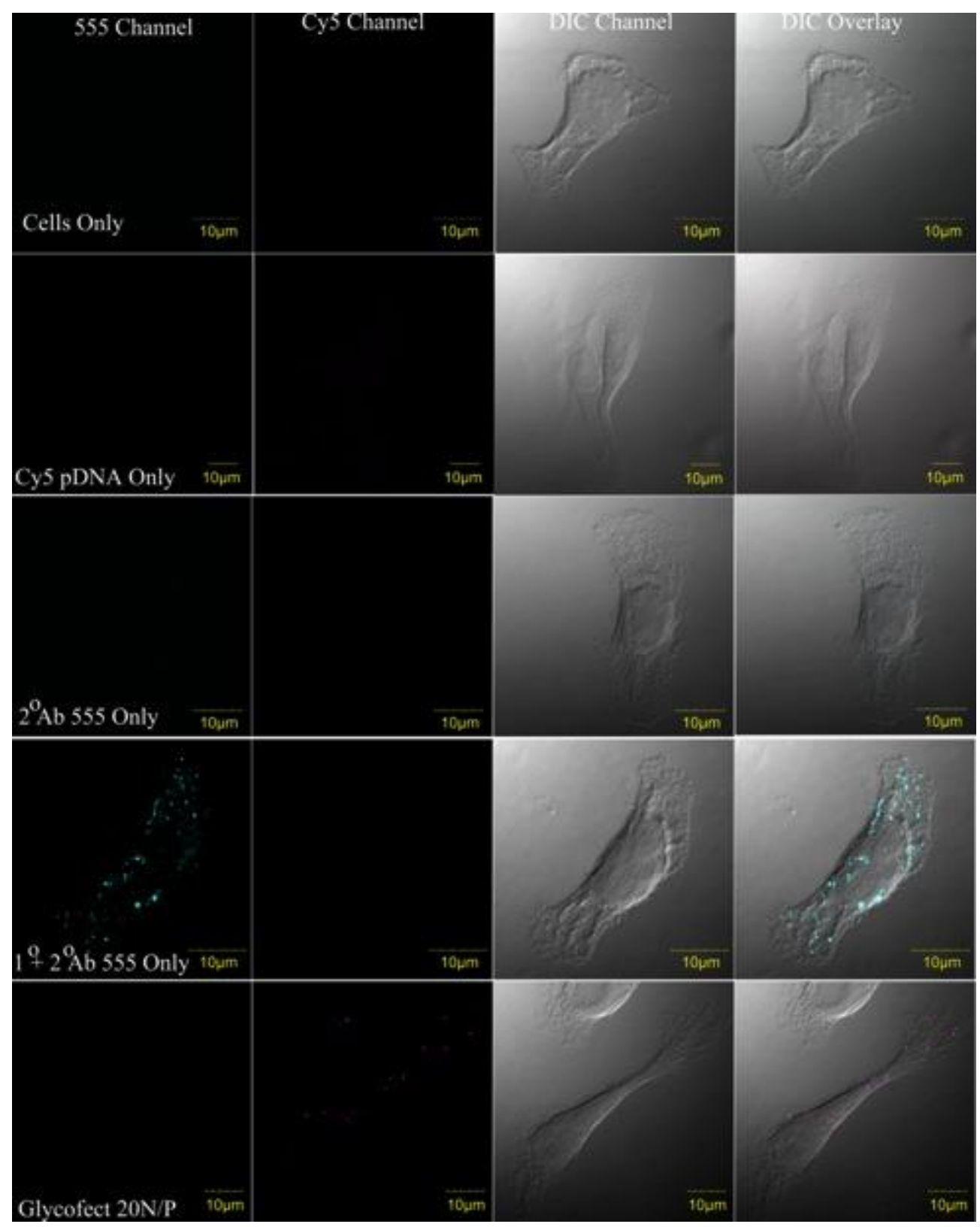

Figure S4. Additional controls for fixed cell imaging using non-sequential imaging. Note: Cells only: untreated HeLa cells only. Cy5-pDNA only: cells treated with naked Cy5-pDNA only. $2^{\circ} \mathrm{Ab} 555$ only: secondary antibody 555 only. $1^{\circ}+2^{\circ} \mathrm{Ab} 55$ only: caveolin- 1 primary antibody and secondary antibody 555 (pseudo colored cyan). Glycofect at N/P $=20$ : HeLa cells were treated (i.e. transfected) with Glycofect polyplexes. These polyplexes were made by adding Glycofect polymer at $20 \mathrm{~N} / \mathrm{P}$ to fluorescently labeled plasmid Cy5-pDNA at $0.02 \mu \mathrm{g} / \mu \mathrm{L}$ (pseudo colored magenta). Confocal microscope: Olympus FluoView FV1000 IX2. Objective: 100x, NA: 1.3, oil immersion. Excitation lasers: $543 \mathrm{~nm}$ for Alexa Fluor 555 and $633 \mathrm{~nm}$ for Cy5. 


\section{Live-cell imaging}

A series of live-cell images that visualize GFP-actin are shown in Figure S5. A glycofect polyplex is observed moving along actin. This suggests that after a polyplex traffics from the filopodial base to the cell surface, it may traffic along the edge of a cell.

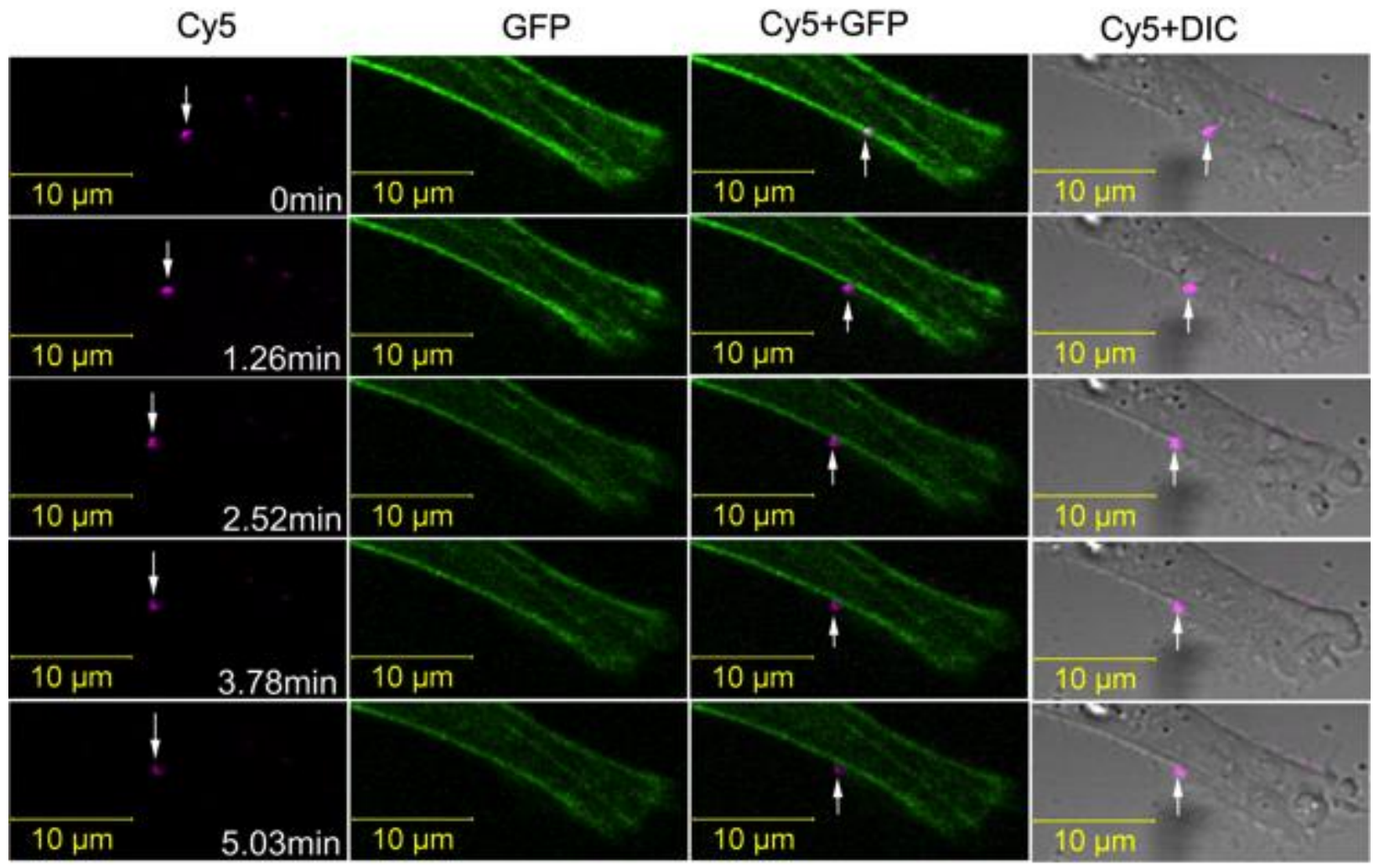

Figure S5. Low magnification time series images of polyplex moving along actin at the edge of a HeLa cell. Column 1 for Cy5: Polyplex was formulated with Glycofect at 20 N/P and Cy5pDNA. Polyplex is pseudo-colored 'magenta', Column-2 for GFP: HeLa cells were stably expressing GFP-actin. Actin is pseudo-colored 'green', Column-3 for Cy5+GFP: Overlay showing polyplex trafficking along actin, Column-4 for Cy5+DIC: Overlay showing polyplex trafficking along the edge of cell boundary. Note: Live cell images were taken by Zeiss LSM Meta 510 confocal microscope (Virginia Tech). Objective: 100x, NA: 1.45, oil immersion. Excitation lasers: $488 \mathrm{~nm}$ for GFP and $633 \mathrm{~nm}$ for Cy5 (see Supporting Information Video 9). 


\section{Fixed-cell imaging}

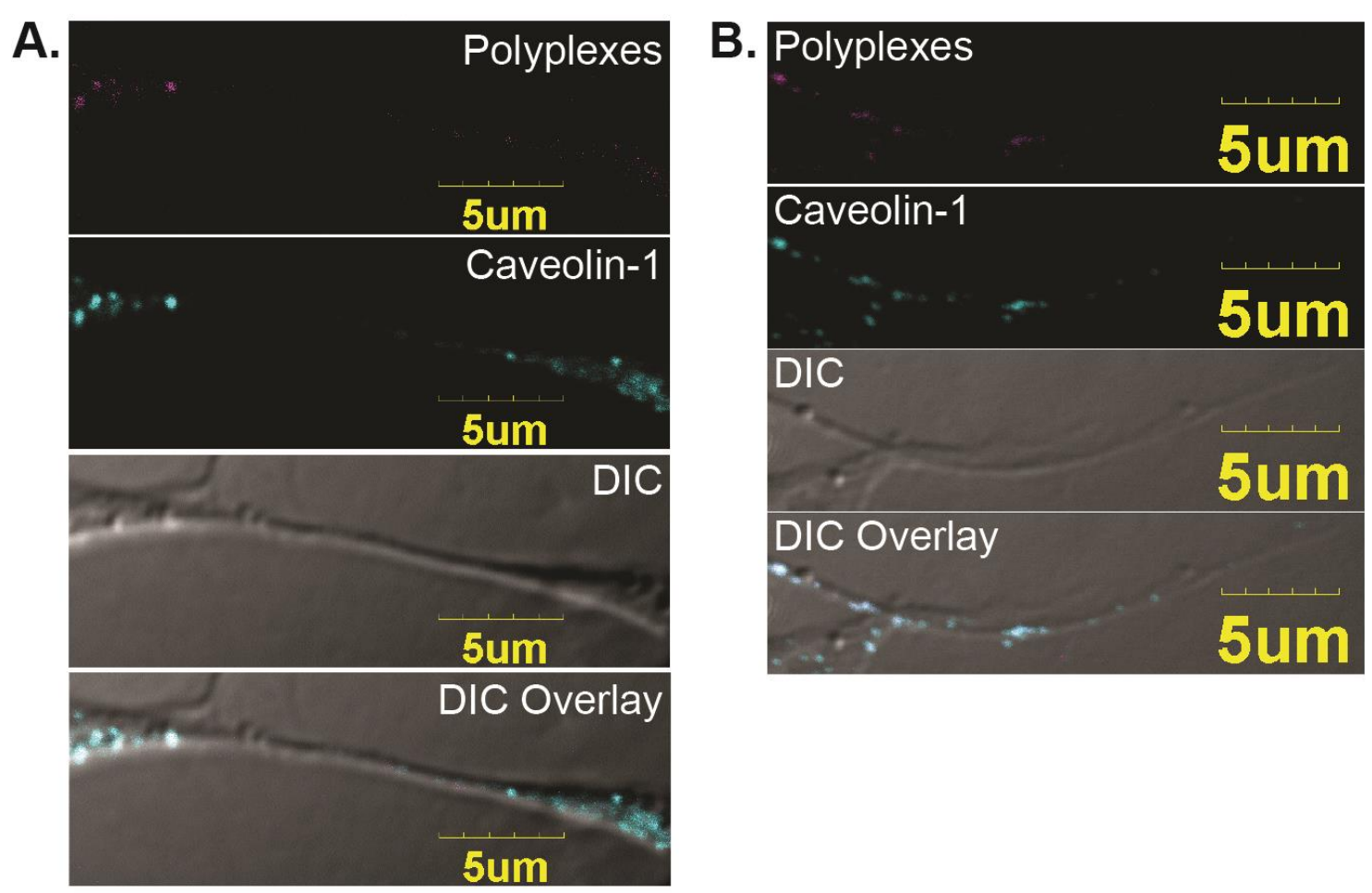

Figure S6. Fixed-cell images of endocytic vesicles trafficking along filopodia in HeLa-GFPActin cells. (A) and (B) Caveolae labeled with anti-caveolin-1 primary antibody (pseudo-colored cyan). Glycofect polyplexes $(\mathrm{N} / \mathrm{P}=20)$ are pseudo colored magenta. 


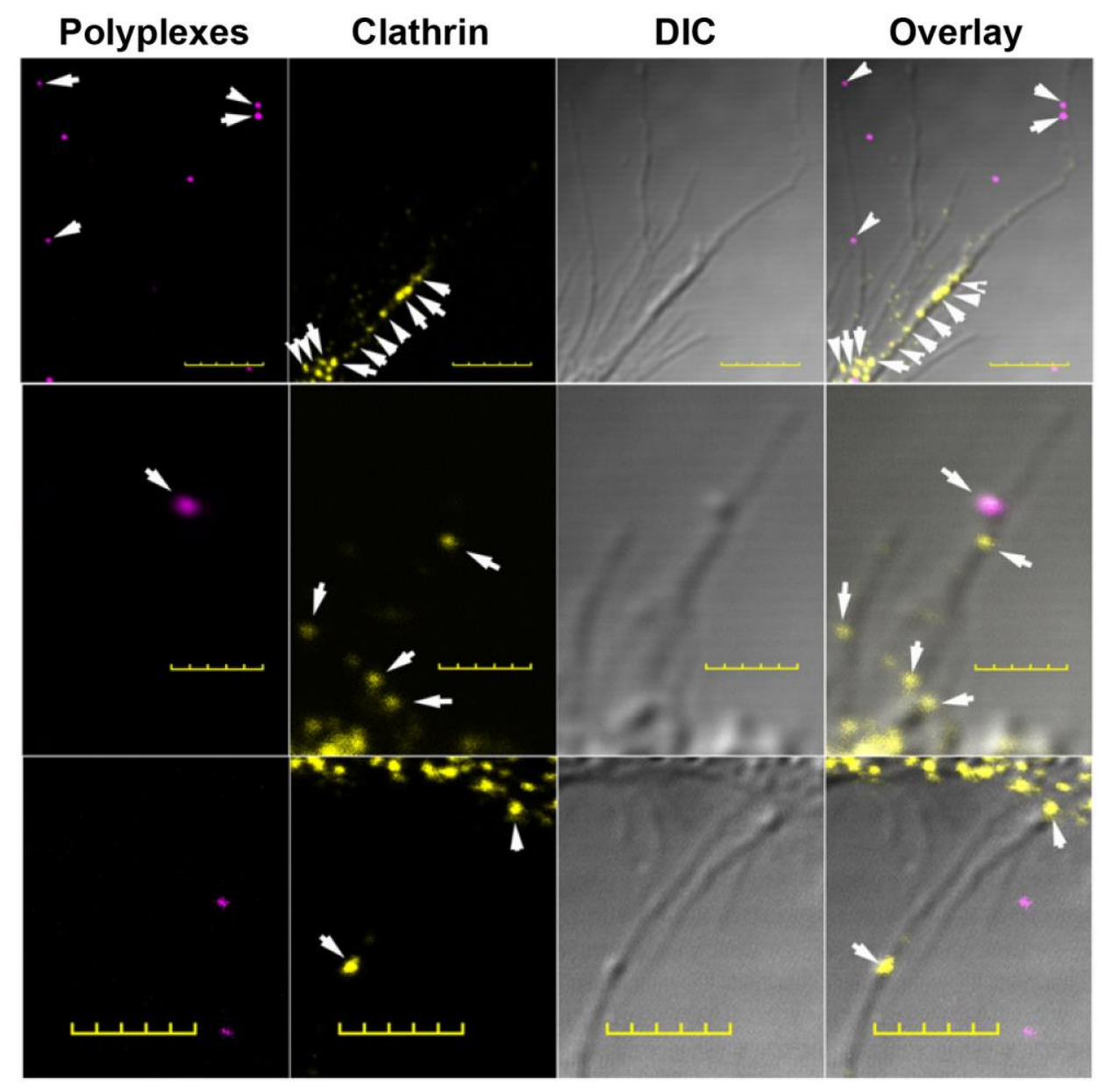

Figure S7. Fixed-cell images of endocytic vesicles trafficking along filopodia in a HeLa-GFPActin cell. Clathrin labeled with an anti-clathrin heavy-chain primary antibody (pseudo-colored yellow). Glycofect polyplexes $(\mathrm{N} / \mathrm{P}=20)$ are pseudo-colored magenta. Scale bars represent 5 $\mu \mathrm{m}$. 


\section{Statistical analysis}

Table S1: Single factor analysis of variance (ANOVA) of velocities of $\mathrm{N}=19$ vesicles. The 'Count' indicates the number of images a vesicle was tracked, 'Average' indicates the average velocity of a vesicle. The $\mathrm{p}$-value $<0.05$ indicates that the velocity of at least one of the vesicles is significantly different.

\begin{tabular}{|c|c|c|c|c|}
\hline Groups & Count & Sum & Average & Variance \\
\hline Vesicle_1 & 19 & 0.399421 & 0.021022 & 0.000258 \\
\hline Vesicle_2 & 4 & 0.166923 & 0.041731 & 6.67E-05 \\
\hline Vesicle_3 & 7 & 0.245079 & 0.035011 & $9.84 \mathrm{E}-05$ \\
\hline Vesicle_4 & 4 & 0.138019 & 0.034505 & 0.000183 \\
\hline Vesicle_5 & 5 & 0.146335 & 0.029267 & 4.69E-05 \\
\hline Vesicle_6 & 15 & 0.335784 & 0.022386 & 0.000101 \\
\hline Vesicle_7 & 7 & 0.195269 & 0.027896 & 7.3E-05 \\
\hline Vesicle_8 & 9 & 0.3424 & 0.038044 & 0.000348 \\
\hline Vesicle_9 & 10 & 0.403613 & 0.040361 & 4.52E-05 \\
\hline Vesicle_10 & 4 & 0.209725 & 0.052431 & $9.37 \mathrm{E}-06$ \\
\hline Vesicle_11 & 6 & 0.293503 & 0.048917 & 0.000452 \\
\hline Vesicle_12 & 152 & 18.53963 & 0.121971 & 0.006458 \\
\hline Vesicle_13 & 9 & 0.082528 & 0.00917 & $2.96 \mathrm{E}-05$ \\
\hline Vesicle_14 & 4 & 0.030216 & 0.007554 & $3.26 \mathrm{E}-05$ \\
\hline Vesicle_15 & 6 & 0.075993 & 0.012666 & $9.2 \mathrm{E}-05$ \\
\hline Vesicle_16 & 2 & 0.087029 & 0.043515 & 0.000168 \\
\hline Vesicle_17 & 6 & 0.21626 & 0.036043 & 0.00014 \\
\hline Vesicle_18 & 3 & 0.088204 & 0.029401 & 0.000407 \\
\hline Vesicle_19 & 1 & 0.027905 & 0.027905 & $\mathrm{~N} / \mathrm{A}$ \\
\hline
\end{tabular}


ANOVA

\begin{tabular}{lcrcccc}
\hline Source of Variation & $S S$ & $d f$ & $M S$ & $F$ & P-value & F crit \\
\hline Between Groups & 0.602088 & 18 & 0.033449 & 8.572387 & $6.76 \mathrm{E}-18$ & 1.644602 \\
Within Groups & 0.991104 & 254 & 0.003902 & & & \\
& & & & & & \\
Total & 1.593192 & 272 & & & & \\
& & & & & & \\
\hline
\end{tabular}




\section{SI Video Legends}

Note: The polyplexes in each video were prepared at $\mathrm{N} / \mathrm{P}=20$ using Glycofect polymer and Cy5-labeled plasmid DNA (magenta color).

Supporting Information Video 1: Live cell confocal imaging of a vesicle moving along a filopodium in a HeLa-Actin-GFP cell in Opti-MEM at $37{ }^{\circ} \mathrm{C}$. The vesicle can be initially observed moving along filopodium and later moving into the receding lamellipodium. Image capture rate: 1 minute per frame. Video playback speed: 1 frame/second.

Supporting Information Video 2: Live-cell confocal imaging of a nanocomplex trafficking along actin at the edge of a HeLa-Actin-GFP cell in Opti-MEM at $37{ }^{\circ} \mathrm{C}$. Scan speed: 1 frame per 12.6 seconds. Video playback speed: 2 frames per second.

Supporting Information Video 3: A filopodium detects an immobile polyplex in the extracellular space and this recognition causes the cell lamellipodia to advance toward the polyplex. Image capture rate: 30 seconds per frame. Video playback speed: 2 frames per second.

Supporting Information Video 4: Live cell confocal imaging of polyplex along a filopodium in HeLa-Actin-GFP cells in Opti-MEM at $37{ }^{\circ} \mathrm{C}$. The polyplex can be observed moving along a filopodial bridge over extracellular space from a cell on the left towards the cell on the right. Image capture rate: 30 seconds per frame. Video playback speed: 1 frame per second.

\section{References}

(1) Liu, Y.; Wenning, L.; Lynch, M.; Reineke, T. M. New Poly(D-glucaramidoamine)s Induce DNA Nanoparticle Formation and Efficient Gene Delivery into Mammalian Cells. J. Am. Chem. Soc. 2004, 126 (24), 7422-7423.

(2) Liu, Y.; Reineke, T. M. Hydroxyl Stereochemistry and Amine Number within Poly(glycoamidoamine)s Affect Intracellular DNA Delivery. J. Am. Chem. Soc. 2005, 127 (9), 3004-3015.

(3) Liu, Y.; Reineke, T. M. Poly(glycoamidoamine)s for Gene Delivery: Stability of Polyplexes and Efficacy with Cardiomyoblast Cells. Bioconjug. Chem. 2006, 17 (1), 101108.

(4) Lee, C.-C.; Liu, Y.; Reineke, T. M. General Structure-Activity Relationship for Poly(glycoamidoamine)s: The Effect of Amine Density on Cytotoxicity and DNA Delivery Efficiency. Bioconjug. Chem. 2008, 19 (2), 428-440.

(5) Liu, Y.; Reineke, T. M. Poly(glycoamidoamine)s for Gene Delivery. Structural Effects on Cellular Internalization, Buffering Capacity, and Gene Expression. Bioconjug. Chem. 2007, 18 (1), 19-30. 
